Alexander

Filipović

\title{
Neue Medienkompetenz und Beteiligungsgerechtigkeit Herausforderungen für die Medien- und Kommunikationsethik ${ }^{1}$
}

\section{Einleitung}

Anfang des Jahres 1970 wurde das „Kassettenfernsehen“ geboren. Der Publizist Dieter E. Zimmer betätigte sich als Geburtshelfer und rief aus: „Ein neues Medium kommt auf die Welt" (Zimmer 1970). Mit Kassettenfernsehen ist der Videorecorder gemeint und dabei geht es im Jahr 1970 darum, "daß der Zuschauer sich unabhängig von den Fernsehprogram. men der Rundfunkanstalten machen soll, indem jeder sich seine eigenen Programme zusammenstellt" (Zimmer 1970, S. 7). Nicht minder revolutionär der Videotext: Allein die aufgeregten Debatten darüber, ob das neue Medium als Rundfunk zu bezeichnen sei, verrät die Verunsicherung.

Ein letztes Beispiel aus der ersten Generation der „Neuen Medien“: das Kabelfernsehen. Der bereits zitierte Dieter E. Zimmer beschreibt 1970 hellsichtig die Möglichkeiten eines flächendeckenden Kabelfernsehens: Damit wäre eine Zeit angebrochen, in der ,jeder von seinem Fernsehapparat aus große zentrale Cinematheken oder Videotheken anwählen kann, um sich die dort archivierten Filmprogramme auf seinen Bildschirm überspielen zu lassen; wo er aus Datenbanken Informationen, aus Bibliotheken auf Video-Speicher übertragene Buchinhalte abrufen und zu Hause oder am Arbeitsplatz auf dem Bildschirm lesen [...] kann; und wo sogar die Tageszeitungen ihren Abonnenten von den Presseverlagen per Kabel ins Haus geschickt werden" (Zimmer 1970, S. 7).

In der Beurteilung der Situation scheiden sich natürlich die Geister. Die einen sehen in bewahrend-kulturpessimistischer Perspektive erneut den Untergang des Abendlandes kurz bevorstehen. Hans Bausch beschreibt dies 1985 und konstatiert, dass dies bei jedem neuen Medium der Massenkommunikation üblich ist: „Wie jedem neuen Medium der Massenkommunikation schlug auch dem Fernsehen eine Besorgniswelle entgegen, die stark kulturpessimistische Züge trug. Besorgte Stimmen sagten das Ende aller Aktivitäten in der Gesellschaft voraus: das Ende

1 Überarbeiteter Text des Vortrags „Herausforderungen für die Kommunikations- und Medienethik“, gehalten beim Symposium „Zukunlt der Medien - Medien der Zukunft" am 22.11.2006 im Presseclub Nürnberg, veranstaltet durch den Mediencampus Bayern e.V., Schule für Rundfunktechnik Nürnberg und den Presseclub Nürnberg. Der Vortragsduktus wurde behutsam angepasst. 
aller Vereine, Konzerte, Theater, Galerien, der Buchproduktion, der Presse und des Radios" (Bausch 1985, S. 250).

Die andere Perspektive ist fast hemmungslos euphorisch aufgrund der vielen neuen Möglichkeiten. Sie sehen die Befreiung von dem Diktat des Programms, erwarten eine Gesellschaft ohne Informationslïcken, interpretieren neue Medien als zwangsläufig demokratiefördernd und erkennen die technischen Möglichkeiten als wahren Fortschritt, weil sie die begrenzten Möglichkeiten des Menschen zu überschreiten helfen.

Dieser Blick in die deutsche Situation der 70er Jahre des 20. Jahrhunderts (vgl. Fromm 2006) ist auch für die medienethische Perspektive sehr heilsam. Wenn nämlich in ähnlich kulturpessimistischer oder euphorisch-fortschrittsoptimistischer Weise die momentane Situation des Medienwandels kommentiert wird, besteht die Gefahr, entweder das humane Potenzial dieses Wandels zu übersehen oder die ethisch relevanten Probleme nicht in den Blick zu bekommen. Für die folgenden Überlegungen ist von vorneherein darauf $z u$ achten, nicht in das Wasser zwischen der Scylla der Neue-Medien-Euphorie und der Charybdis des Kulturpessimismus zu kommen, sondern in anderen Wassern gefahrloser und vor allem ertragreicher $z \mathfrak{u}$ fischen.

Worin aber nun die spezifischen Herausforderungen neuer Medien und ihrer Techniken aus der Sicht eines Medien- und Kommunikationsethikers bestehen, lässt sich erst sagen, wenn das Verständnis von Medienund Kommunikationsethik explizit gemacht wird. Denn die Herausforderungen stellen sich aus der Sicht eines Medieninformatikers, einer Chefredakteurin im Printbereich, einer Medientechnikerin oder eines Medienund Kommunikationsethikers anders dar - und: Es ist ja auch durchaus nicht klar, was Ethik überhaupt ist; jedenfalls herrscht alltagssprachlich eine ziemliche Begriffverwirrung. Im Folgenden wird also zunächst ein spezifisches Verständnis der sozialethischen Beschäftigung mit Medien und öffentlicher Kommunikation dargelegt. Ausgehend von diesem Verständnis können die Herausforderungen des derzeitigen Medienwandels skizziert werden.

\section{Medien- und Kommunikationsethik - was ist das eigentlich?}

\subsection{Moral und Ethik}

Im Prinzip geht es bei Moral um die Unterscheidung von gut und schlecht oder von gut und böse. Von der Wortbedeutung her ist Moral ungefähr gleichbedeutend mit dem Begriff der Sitte. Dieser Zusammenhang verweist auf den Umstand, dass die Unterscheidung von gut und schlecht 
immer etwas mit dem allgemeinen Verständnis zu tun hat, was als gut und was als schlecht anzusehen ist. Bei der Moral geht es nicht um rein sachliche Dinge, also etwa: "Ist es gut oder schlecht, dass es regnet?", auch nicht um technische Dinge, also etwa: „Ist es gut oder schlecht, dass jetzt Bildsignale auf kleine Endgeräte übertragen werden können?", oder um eine Gleichsetzung von „funktioniert" und „gut" oder "kaputt" und "schlecht", sondern es geht um Lebens-Gestaltung, Lebens-Sinn und Gerechtigkeit, um den Menschen, um die Achtung vor dem Menschen und um seine Anerkennung, kurz: um das Humane.

Moral hat daher etwas mit Verbindlichkeit zu tun, also mit einer bestimmten Geltung von Kriterien, nach denen etwas gut oder schlecht ist und die anzuerkennen sind. Zusammenfassend kann man definieren: „Moral ist die Einsicht in die Verbindlichkeit von Sinngehalten des Lebens, die man anerkennen muss, wenn man dieses Leben so führen will, dass es der Würde eines jeden Menschen entspricht" (Mieth 2004, S. 21).

Ethik nun ist etwas ganz anderes als Moral. Die Begriffe werden im Alltagsverständnis zwar weitgehend synonym gebraucht, aber es erscheint besonders für das Thema der Medienethik wichtig zu sein, beides $z \mathfrak{u}$ trennen. Ethik begreift die Moral als ihren Gegenstand. Sie erscheint als eine besondere Denkform, nämlich eine reflektierende, eine nachdenkende, eine theoretische Denkform. Ethik ist also eine Theorie der Moral, und sie wird notwendig, wenn die Moral selber strittig wird, wenn es also nicht klar ist, was verbindlich ist, was ein Gut oder ein Wert sein und was in Bezug auf Gerechtigkeit und gutes Leben gelten soll. Insofern lässt sich Ethik verstehen als "Nachdenklichkeit über strittige Moral“ (Mieth 2004, S. 24). Damit ist sie nicht nur und vor allem die Orientierung selber, sondern der Streit über Orientierungen.

\subsection{Medienethik als Streit über Orientierungen}

Die Medien - und Kommunikationsethik beteiligt sich am Streit ïber Orientierung im Bereich der Medien: Sie entdeckt und dokumentiert die Debatte über Wertungen und Orientierungen, systematisiert den Streit, überführt ihn in argumentativ zugängliche Zusammenhänge und ver. sucht ihn kommentierend zu moderieren und mit normativen Vorschlä. gen voranzubringen.

Wenn Medienethik in diesem Sinne ein Streit über Orientierungen ist, die prekär geworden sind, dann ist dieses Verständnis von Medienethik weit entfernt von einer moralisierenden Skandalethik. Diese entzündet sich am Konflikt und ist immer im Recht, weil jeder einsieht, dass der Skandal in der Tat skandalös ist. Erinnert man sich noch an die Empö- 
rung und Aufregung um Big-Brother (vgl. z.B. Bohrmann 2000 und Schweer et al. 2002) oder an die scharfe Auseinandersetzung um die so genannte Benetton-Schockwerbung (vgl. z.B. Jäckel/Reinhardt 2003)? Es besteht kein Anlass zu sagen, dass die Auseinandersetzungen in diesen beiden Fällen umsonst oder gar schädlich gewesen wären oder dass hier gar keine medienethisch bedeutsamen Fälle vorliegen. Gerade im Fall der Benetton-Werbung gipfelte die Auseinandersetzung in einem höchst interessanten Urteil des Bundesverfassungsgerichts, das darin die Kommunikationsfreiheit höher eingeschätzt hat als die Gesetze gegen den unlauteren Wettbewerb. Aber Medienethik darf, soll sie eine gewisse Wirksamkeit entfalten, nicht als moralisierende Skandalethik verstanden werden, die immer nur dann und übrigens immer zu spät aus den Löchern kommt, wenn gesellschaftlicher Orientierungsbedarf entsteht. In diesem Klima verlangen die Leute, dass jetzt endlich mal jemand mit Autorität sagt, dass das ja unmoralisch ist, was in den Medien und mit den Medien so passiert.

Medienethik, die der Versuchung erliegt und so auftritt, wird über kurz oder lang als nörgelnd, besserwisserisch und moralisierend wahrgenommen. Wenn moralisches Argumentieren in solchen Fällen derart enthusiastisch unternommen wird, die öffentliche Arena sozusagen zu einem großen Stammtisch wird, hat die Medienethik durchaus die tatsächlich genuin ethische Aufgabe, vor einer solchen Moral, oder besser: vor einem solchen Moralgebrauch, zu warnen. ${ }^{2}$ Vielleicht liegt hier die größte Herausforderung einer zeitgemäßen Medien- und Kommunikationsethik. Dieses Selbstverständnis befreit die Medienethik dabei nicht davon, auf drängende Moralprobleme aufmerksam zu machen; entscheidend bleibt aber der Modus, in dem das geschieht.

Themen der Medien- und Kommunikationsethik sind vor allem gesellschaftliche Strukturen und Institutionen (z.B. Medienrecht, Rundfunksystem, das Internet, die Zeitungslandschaft, Programme), Rollenerwartungen (z.B. „der Journalist“, „der Werbefachmann“, „der Blogger“, „der Leser"), historische Vorgaben (z. B. die politische Verzweckung der öffentlichen Kommunikation im nationalsozialistischen Deutschland) und Möglichkeitsbedingungen kommunikativer Beteiligung (z. B. Medienkompetenz, ökonomische Ressourcen, Verstehensleistungen). Diese und andere sozialen Phänomene beeinflussen und bestimmen unser Handeln, auch unser moralisches Handeln. Sie sind zwar in gewissem Sinne eigendynamisch und unterliegen nicht persönlichen Verantwortlichkeiten,

2 Vgl. Luhmann 1991, S. 41. Zu den Möglichkeiten einer Medienethik, die sich auf Luhmanns Ethikverständnis und eine systemtheoretische Analyse einlässt, vgl. Filipović 2003. 
aber sie sind auch nicht naturgegeben, sondern sind wandelbar, und vor allem können und müssen sie gestaltet werden.

Medienethik hat diese Gestaltung von gesellschaftlichen Strukturen zum Thema und betreibt damit, jedenfalls in pluralen demokratischen Gesellschaften, kommunikationspolitische Beratung. Medien- und Kommunikationsethik ist das Nachdenken über strittige Moral die Gestaltung des sozialen Bereichs betreffend, der mit Medien und öffentlicher Kommunikation bezeichnet wird. Medienethik entdeckt solche Strukturen und bringt sie mit den moralischen Themen Lebens-Gestaltung, LebensSinn, Gerechtigkeit, Menschlichkeit, Achtung vor dem Menschen und seine Anerkennung zusammen. ${ }^{3}$

\subsection{Medienethik 2.0?}

Die aus der Informatik stammende Versionskennzeichnung „2.0" für eine grundlegend veränderte neue zweite Version einer Software hat mittlerweile nicht nur das Internet in Form des Ausdrucks „Web 2.0" erreicht, sondern man spricht gar von „Ethik 2.0“ (vgl. Filipović 2007b). Bedarf die Ethik und darin die Medien- und Kommunikationsethik eines Updates? Müssen wir uns um eine Medienethik 2.0 bemühen?

Das Schlagwort Web 2.0 ist zwar ein in ökonomischen Zusammenhängen $z \mathfrak{u}$ oft gebrauchtes Modewort, macht aber dennoch darauf aufmerksam, dass das Word Wide Web eine neue Qualität erreicht und Kommunikation hier in neuer Weise verwirklicht wird. Die Menschen erfahren normative Ansprïche an ihr Kommunizieren und Handeln vermehrt in diesen spezifischen Situationen des "social web"; ihnen ist die Gestaltung des Lebens und die Bewältigung dieses Projektes in konkreten Fällen, sozusagen „wenn es darauf ankommt", aufgegeben. Diese Situationen und Kontexte, also die neuen Medien, die Veränderung etablierter Publikationen, die neuen Inhalte und Formen, das "social web" und das Web 2.0, die "social software" usw. gilt es medien- und kommunikationsethisch im Blick $z \mathfrak{u}$ behalten, ohne dass dabei den Situationen und Kontexten eine moralisch begründende Funktion zuerkannt werden kann. Andernfalls würde man den Kontakt zum dynamischen Gegenstand der Medienethik verlieren oder den Anspruch nicht aufrecht erhalten können, normativ $z \mathfrak{u}$ argumentieren. Insofern ist die Medienethik also updatefähig und -bedürftig.

3 Die medienethische Literatur ist sehr vielschichtig. Für einen systematischen Einstieg in die Thematik eignen sich z.B. Karmasin 1999, Debatin 2002, Heesen 2002 Hausmanninger 2005 und Funiok 2007. Die deutschsprachige medienethische Szene ist verknüpft im Netzwerk Medienethik (vgl. www.netzwerk-medienethik.de). 


\section{Medientechnik und neue Medien als Herausforderung der Medien- und Kommunikationsethik?}

Mit dieser Vorstellung von Medien- und Kommunikationsethik können nun diejenigen Entwicklungen der Medien- und Kommunikationswelt identifiziert werden, die als Herausforderung zu begreifen sind. Selbstverständlich kann nur eine Auswahl präsentiert werden. Nicht Skandale oder die behauptete Unmoral der Medien sind dabei der Ausgangspunkt, sondern es wird versucht, Probleme im Umfeld von Medien und Kommunikation zu entdecken, die mit Gerechtigkeit und gutem Leben zusammenhängen.

Dafür kann von den bereits erwähnten Beispielen ausgegangen werden: Das „Kassettenfernsehen“ führt erstens die heute sehr aktuelle Herausforderung der vermehrt möglichen Selbstgestaltung und Selbstzusammenstellung von medialen Inhalten ein (Stichwort Webcasts, Newsreader, RSS-Feeds). Das Beispiel Kabelfernsehen verweist in der zitierten hellsichtigen Interpretation von 1970 zweitens auf die Herausforderungen, die sich aus einem umfassenden und unüberschaubaren Angebot an Informationen und Wissensbeständen ergeben. Klar ist, dass beide Beispiele heute im Zusammenhang gesehen werden müssen.

\subsection{Selbstgestaltung und Selbstzusammenstellung von medialen Inhalten als Herausforderung}

Der Videorekorder steht am Anfang der Emanzipation des Zuschauers vom Programm - jedenfalls vom Prinzip her. Heute sind die technischen Möglichkeiten weit größer. Mit der Digitalisienung audiovisuellen Materials, variableren Aufzeichnungsmethoden und breiteren Übertragungsmöglichkeiten geht auch die Möglichkeit einher, sich ganz dem Zwang zur Programmierung bzw. Sequenzierung von Sendungen $z u$ entziehen. Sendeschemata in Form von Fernseh- und Hörfunkprogrammen verlieren ihren Sinn dann, wenn durch eine Pluralisierung der Lebensgewohnheiten und der Tagesrythmen gar nicht mehr davon ausgegangen werden kann, dass Zielgruppen zu einer bestimmten Zeit die Möglichkeit zum Fernsehschauen haben. So weit ist es noch nicht, aber die Nachfrage nach zeitunabhängigem Fernsehschauen und Radiohören wächst. Man spricht von einer „Entlinearisierung“ des Fernsehens und des Hörfunks (vgl. auch Gehlen 2007).

Der Erfolg von Online-Videoportalen (z.B. „YouTube“) oder sogenannten Podcasts zeigt dies deutlich. Auf etlichen Videoportalen im Internet ist audiovisuelles Material jederzeit einsehbar. Es ist übrigens ein Irrtum zu glauben, dort wären nur seichte Amateurvideos anzuschauen; mit ein 
wenig Suche finden sich dort Interviews und Dokumentationen auch für wissenschaftlich Interessierte. Podcasts und RSS-Feeds ihrerseits revolutionieren die vormals so prägenden Sende- und Empfangsverhältnisse: Mit einem Podcast abonniert man bestimmte Inhalte via Internet, egal ob nur Ton oder auch audiovisuelles Material, synchronisiert ein entsprechendes Gerät mit dem Computer und schon kann man das bevorzugte Programm hören, wenn man dafür Zeit hat. Neue mobile Endgeräte kommen diesem Wunsch nach Unabhängigkeit entgegen, wobei nicht nur die Unabhängigkeit von Programmschemata augenfällig ist, sondern auch die Unabhängigkeit von zentral und immobil installierten Endgeräten. Liveübertragungen wie Fußballspiele, Konzerte oder "Wetten dass" sind über Handy-TV oder den DVB.T Empfänger am Notebook auch am Badesee oder im Café zu erleben.

Ähnlich verhält es sich mit Textinhalten. Warum soll ich eine Tageszeitung abonnieren, wenn ich per RSS-Newsreader (um eine denkbare Kombination beispielhaft $\mathrm{zu}$ präsentieren) die wichtigsten politischen Nachrichten und Kommentare aus der „Süddeutschen“, den Sport aus dem Kicker, und die Wirtschaft aus der "FAZ" und das Feuilleton aus der Frankfurter Rundschau übersichtlich aufbereitet auf einer Internetseite (oder in der Zukunft auf elektronischem Papier) dargestellt bekomme. Hinzu kommen meine Lieblingsweblogs und Feeds von Freunden, Kollegen und innovativ schreibenden Bloggern, und der Tag beginnt mit dem persönlichen Nachrichtenmix. Zum gemütlichen „echten“ Zeitungslesen reicht der Sonntagmorgen mit der Sonntagszeitung.

Dieser noch sehr zaghafte Umstieg vom Push-Prinzip massenmedialer Kommunikation hin zu einem Pull-Prinzip, nach dem sich Menschen gemäß individueller Gesichtspunkte den Mediamix aussuchen und auf den Computer oder das Handy „downloaden“, stellt zunächst einmal Programmdirektoren und Zeitungsmacher vor das Problem, überhaupt noch ein Programm oder eine Zeitung, also linearisierten und für andere ausgewählten Content anzubieten, das oder die den Menschen gefällt. Ich erkenne hier tatsächlich eine gewisse Verantwortung zum Beispiel der öffentlich-rechtlichen Rundfunkanstalten, diesen Trends nicht hinterherzulaufen, sondern von Anfang an die veränderten Gewohnheiten mit qualitativ hochwertigen Produkten $z \mathfrak{u}$ bedienen.

Die Freiheit und die Autonomie der Menschen im Umgang mit medialen Inhalten scheint mir aber die noch bedeutsamere medien- und kommunikationsethische Herausforderung zu sein. Die aktive Rolle kommt vermehrt dem Zuschauer zu. Das können Menschen nicht von selbst. Wer bringt es ihnen bei? Wer hilft bei der Auswahl, wer vermittelt grundlegende Kompetenzen, mit denen eine Auswahl gut getroffen werden kann, und wer hilft bei der Bedienung der dazu notwendigen Geräte? Sollen die 
Freiheitspotentiale dieses Medienwandels realisiert werden, so geschieht dies sicherlich nicht von selbst, vor allem nicht für jene Menschen, die nicht mit solchen Möglichkeiten heranwachsen.

Zudem besteht die Gefahr, dass ein gewisser Teil der Bevölkerung nicht die Möglichkeit hat, diese Freiheitspotentiale zu heben: Geräte kosten Geld, Inhalte erfordern Verstehensvoraussetzungen. Was, wenn beides für Teile der Bevölkerung knapp ist? Ist ein sich, wie jüngst festgestellt, stabilisierender gesellschaftlicher Ausschluss bestimmter Schichten nicht ein klarer Fall sozialer Ungerechtigkeit und hat er nicht auch mit Beteiligung an Medien und öffentlicher Kommunikation zu tun? Wenn die Beteiligung auch und gerade an neuen Formen öffentlicher Kommunikation eine Gerechtigkeitsfrage ist, wird Medien- und Kommunikationspolitik zusammen mit Medienpädagogik und Medienandragogik zu einer sozialpolitischen Angelegenheit.

\subsection{Zum umfassenden und unüberschaubaren Angebot an Informationen und Wissensbeständen}

Die zitierte Prognose zu den neuen Möglichkeiten des Kabelfernsehens ist heute wahr geworden. Sicherlich in ganz anderer Weise, als 1970 absehbar war, aber immerhin: Heute kann man sich von jedem Computer mit Netzzugang in die Heimatbibliothek einklicken, jedes Informationsangebot suchen und finden; viele Studenten und Studentinnen verlassen für eine Seminararbeit nicht mehr das Wohnheimzimmer, um in der Bibliothek in Büchern zu blättem, sondern Aufsatzdatenbanken und andere Informationssysteme liefern zu jedem Thema etliche Treffer und zitierbare Originaltexte.

Mit der entsprechenden Ausstattung entfällt auch der Gang in die Videothek: Über den digitalen Satelliten- und Kabelempfang und über die VDSL-Leitung kommen eigens ausgesuchte Filme zu selbst bestimmter Zeit auf den LCD-Bildschirm. Wie unsere Eltern ohne die schnelle Internetrecherche eigentlich ihren Alltag bewältigen, ist uns fast nicht mehr klar. Wenn man beim Gespräch mit der Friseurin feststellt, dass diese, eine junge Frau von 30 Jahren, keine ihr bekannten Zugriffsmöglichkeiten auf einen Computer hat, der mit dem Internet verbunden ist, dann ist man erschrocken von der plötzlich in den Blick kommenden Möglichkeit, dass da wohl noch weitere in der Gesellschaft zu finden sind, die von diesen Möglichkeiten ausgeschlossen sind.

Wir leben in der Wissensgesellschaft. ${ }^{4}$ Sicherlich ist das ein $\mathrm{zu}$ oft gebrauchtes und schlagwortartig verwendetes Wort, und der Begriff lei-

4 Vgl. im Folgenden zur Medienethik in der Wissensgesellschaft Filipovic 2007a. 
det darunter, ökonomisch einseitig verwendet zu werden und neben $\mathrm{Be}$ griffen wie Erlebnisgesellschaft, Risikogesellschaft und Wissenschaftsgesellschaft zu einer Charakterbeschreibung moderner Gesellschaft unter anderen $z \mathfrak{u}$ verkommen. Wenn man aber von einer mengentheoretischen Bestimmung des Begriffs absieht (vgl. Heidenreich 2002), entfaltet er meiner Meinung nach eine gewisse analytische Kraft. Mit Wissensgesellschaft ist also nicht gemeint, dass wir heute mehr Wissen als früher produzieren und zur Verfügung haben. Das stimmt sicher, aber mit diesem Argument kann man jedes Zeitalter als Zeitalter des Wissens kennzeichnen: Schließlich wusste man im 19. Jahrhundert auch mehr als im 18. und im 3. Jahrhundert mehr als im 2. vor Christus.

Wenn der Begriff der Wissensgesellschaft Sinn machen soll, dann muss er einen neuartigen Umgang der Gesellschaft mit Wissen kennzeichnen. Dieser neue Umgang beinhaltet, dass wir nun grundsätzlich bereit sind, unsere Erwartungen lembereit zu halten, also jederzeit Lernbereitschaft zu zeigen. Wissensgesellschaft kennzeichnet daher einen neuen Umgang mit dem Nichtwissen. Das betrifft Organisationen ebenso wie Individuen. Sozialisation als die Vermittlung von Individuum und Gesellschaft, die behutsam über Erziehung und Bildung zu erreichen versucht wird, ist in der Wissensgesellschaft also nicht mehr in 8 oder 10 oder 12 Schuljahren zu erreichen, sondern die Teilnahme an der Wis. sensgesellschaft bedeutet, ein lebenslanges Lernen in unserer Lebensgestaltung $z u$ institutionalisieren. Die Anforderung besteht darin, Lernen also nicht als Ausnahmefall in einem bestimmten Lebensabschnitt zu behandeln, sondern auf Dauer zu stellen.

Berücksichtigt man die Bedeutung der Medien und der öffentlichen Kommunikation für die Gesellschaft, dann wird die schon angedeutete Perspektive am Schluss dieser Überlegungen klar: In der Wissensgesellschaft sind alle Formen von Beteiligung nicht ohne den Besitz von bzw. den Zugang zu typischen Wissensformen zu haben. Ohne Wissen keine Beteiligung in und an der Wissensgesellschaft! Wissen (oder besser gesagt: die Fähigkeit $z u$ lernen und eigene Wissensbestände zu erneuern; Lernen-Können) ist in der Gesellschaft aufgrund verschiedener Faktoren sehr unterschiedlich verteilt. Diese Situation führt zu gesellschaftlichen Ungleichheiten, die in der Perspektive der Ethik als Ungerechtigkeit wahrgenommen werden. Menschen, die von der andauernden Veränderung des Wissens überfordert sind, sind z.B. vom Arbeitsmarkt ausgeschlossen. Menschen, die keinen Zugang zu neuem Wissen haben, entscheiden sich vielleicht im Hinblick auf ihre Ernährung oder Gesundheitsvorsorge oder auf Erziehungsmethoden falsch. Kurz: Ein Mangel an Wissen war natürlich auch vor $100 \mathrm{Jahren}$ schon ein Problem. Aber kein so großes wie heute. Denn heute bedeutet Wissensmangel ein 
hochprekäres Unbeteiligtsein und Nichtteilhabenkönnen..$^{5}$ Es gibt keinen Ersatz für aktuelles Wissen und andauernde lebenslange Lembereitschaft.

Dieses soziale Problem, also das Problem der Beteiligung durch Wissen in der Wissensgesellschaft, ist auch das grundlegende Problem einer Ethik, die sich die Frage nach den Medien der Zukunft und der Zukunft der Medien stellt. Die Beteiligung oder Nicht-Beteiligung an den Medien durch alltägliches Fernsehschauen, Zeitunglesen, Radio hören, alltäglichen Internetgebrauch, Podcasts, auch und gerade in der radikal personalisierten Form hat Auswirkungen auf Beteiligungsmöglichkeiten in der Wissensgesellschaft. Wissen wird durch die Medien zur Verfügung gestellt und fallweise angeeignet. Wenn das geschieht, kann man von Wissensvermittlung durch die Medien sprechen. In und durch die Medien geschieht Wissensvermittlung. Und diese Wissensvermittlung durch Medien dient der individuellen alltäglichen Beteiligung an der Wissensgesellschaft. ${ }^{6}$

Gerechte Beteiligungs- und Teilnahmechancen sind Orientierungen zeitgemäßer Medienethik, die unbedingt in die Diskussion einzubringen sind. Das bedeutet eine spezifische Ausrichtung der Medien- und Kommunikationsethik: Weg von einer moralisierenden Medienskandalethik hin zu einer Zuschauer- und Leserorientierung (vgl. Funiok 1996, Veith 2002), die Formen auch populärer Medienangebote nicht gering schätzt, sondern in ihnen die Bedingung der Möglichkeit entdeckt, dass Menschen sich in der modernen Wissensgesellschaft beteiligen können (vgl. Hausmanninger 2005, S. 276f., Filipović 2007a, S. 272-278). Medienund Kommunikationsethik muss die Massenmedien dahingehend untersuchen und beurteilen, inwieweit sie als Möglichkeitsräume der Wissensaneignung genutzt werden können.

Das läuft nicht zuletzt auf ein begründetes Plädoyer für große Anstrengungen zur Ausbildung von Medienkompetenz heraus. ${ }^{7}$ Lebenslanges Lernen darf nicht zu einer Thematik werden, die überall benannt, aber deren Herausforderungen, die damit verbunden sind, nicht angegangen werden. Medienpädagogik mit dem Ziel, dass alle Menschen mit den Medien der Zukunft so umgehen lernen, dass sie sie zum Lemen in jeder

5 Vgl. zur in der modernen Gesellschaft immer drängender werdenden Problematik der Beteiligung, Inklusion und Integration Eckstein/Filipović/Oostenryck 2007.

6 Zur Problematisierung des Begriffs der "Wissensvermittlung" in medienethischen Zusammenhängen vgl. Filipović 2007a, S. 118-131.

7 Vgl. zur Medienkompetenz Groeben/Hurrelmann 2002 und den informierten Überblick mit weiteren Literaturangaben bei Nolda 2002, S. 37-97. Mit der Konzentration auf die frühe Bildung vgl. neuerdings Marci-Boehncke/Rath 2007. 
Lebenslage nutzen können, ist ein Ziel, das übergreifender Anstrengungen bedarf. Im Blick sollten besonders Benachteiligte wie etwa Kinder, Senioren, Menschen mit Lese- und Sprachschwierigkeiten, schlecht ausgebildetete und Langzeitarbeitslose sein. An dieser Stelle wird dann auch das spezifische Profil einer christlichen Medien- und Kommunikationsethik deutlich: Eine christliche Medienethik vertritt keine exklusiven normativen Vorstellungen, sondern sieht sich aus dem christlichen Glauben heraus besonders motiviert, für eine gerechte Gestaltung des Medienbereichs zu streiten. Dabei lässt sie sich von einer biblisch grundgeleg. ten vorrangigen Option für die Armen leiten.

Die Massenmedien selbst können in der Präsentation und der Gestaltung ihrer Inhalte ihren Anteil dazu leisten. Für die Ausbildung von Berufen im Bereich der Medien bedeutet dies, dass lernpsychologische und pädagogische Grundlagen vermittelt und das medienethische Denken eingeübt werden sollten. Um es sicherheitshalber deutlich zu machen: Es geht nicht um mehr Bildungsfernsehen oder darum, aus jedem Journalisten einen Pädagogen zu machen. Es geht auch nicht darum, bestimmte Inhalte als gut oder sinnvoll, andere Inhalte als schlecht oder schädlich zu charakterisieren. Es geht um Ideen, wie ermöglicht werden kann, dass Menschen die Medien der Zukunft für eine Orientierung in der Wissensgesellschaft nutzen können.

Vor allem ist dies eine bildungspolitische Aufgabe: Schule, Universität und Aus- und Weiterbildung sind nicht die Orte, an denen aus der Perspektive eines erhabenen Bildungsideals über die schlechten und niveaulosen Inhalte der Medien und des Internets gewettert werden darf. Schule, Universität und Aus- und Weiterbildung sollten Orte sein, in denen Kinder und Erwachsene lernen, wie man die Freiheitspotentiale neuer Formen öffentlicher, medialer Kommunikation für das lebenslange Lernen in der Wissensgesellschaft nutzt.

\section{Literatur}

Bausch, Hans (1985): Rundfunkpolitische Prognosen von einst und heute. Bemerkungen zur Entwicklung des Fernsehens. In: Schreiber, Erhard/ Langenbucher, Wolfgang R./Hömberg, Walter ( $\mathrm{Hg}$.): Kommunikation im Wandel der Gesellschaft. Festschrift für Otto B. Roegele. 2., rev. u. erw. Aufl. Konstanz, S. 249-254.

Bohrmann, Thomas (2000): Big Brother. Medienethische Überlegungen zu den Grenzen von Unterhaltung. In: Aus Politik und Zeitgeschichte. Beilage zur Wochenzeitung „Das Parlament", Ausgabe B 41-42, 6.10.2000, S. 3-10.

Eckstein, Christiane/Filipović, Alexander/Oostenryck, Klaus (Hg.) (2007): Beteiligung, Inklusion, Integration. Sozialethische Konzepte für die modeme Gesellschaft. Münster. 
Filipovic, Alexander (2003); Niklas Luhmann ernst nehmen? (Un-)Möglichkeiten einer ironischen Ethik öffentlicher Kommunikation. In: Debatin, Bernhard/Funiok, Rïdiger (Hg.): Kommunikations- und Medienethik. Konstanz, S. 83-95.

Filipović, Alexander (2007a): Öffentliche Kommunikation in der Wissensgesellschaft. Sozialethische Analysen. Bielefeld.

Filipović, Alexander (2007b): Ethik 2.0 für das Web 2.0? In: geloggd [Web$\log$, 3. Aug. 2007. Online-Publikation: http://geloggd.alexander-filipovic. de/ 2007/08/03/ethik-20-fur-das-web-20/. Abrufdatum: 10.8.2007.

Fromm, Guido (2000): Vergangene Zukunft - die Neuen Medien der „ersten Generation" in Deutschland. In: Media Perspektiven, Nr. 6, S. 258-265.

Funiok, Rüdiger (1996): Grundfragen einer Publikumsethik. In: Funiok, Rüdiger (Hg.): Grundfragen der Kommunikationsethik. Konstanz, S. 107-122.

Funiok, Rüdiger (2002): Medienpädagogik aus medienethischer Perspektive. In: Paus-Haase, Ingrid/Lampert, Claudia/Süss, Daniel (Hg.): Medienpädagogik in der Kommunikationswissenschaft. Positionen, Perspektiven, Potenziale. Wiesbaden, S. 141-151.

Funiok, Rüdiger (2007): Medienethik. Verantwortung in der Mediengesellschaft. Stuttgart.

Gehlen, Dirk von (2007): Der Terror des Jetzt. Neue Technik verändert unser Kommunikationsverhalten. In: Süddeutsche Zeitung, Nr. 183 vom 10.8. 2007, S. 12.

Groeben, Norbert/Hurrelmann, Bettina (Hg.) (2002): Medienkompetenz. Voraussetzungen, Dimensionen, Funktionen. Weinheim.

Hausmanninger, Thomas (2005): Mediale Öffentlichkeit und Beteiligung. In: Heimbach-Steins, Marianne (Hg.): Christliche Sozialethik. Ein Lehrbuch. Band 2: Konkretionen. Regensburg, S. 254-280.

Heesen, Jessica (2002): Art. Medienethik. In: Düwell, Marcus/Hübenthal, Christoph/Werner, Micha H. (Hg.): Handbuch Ethik. Stuttgart, Weimar, S. 263-268.

Heidenreich, Martin (2002): Merkmale der Wissensgesellschaft. In: BundLänder-Kommission für Bildungsplanung und Forschungsförderung; Bundesministerium für Bildung, Wissenschaft und Kultur/Schweizerische Konferenz der kantonalen Erziehungsdirektoren ( $\mathrm{Hg}$.): Lernen in der Wissensgesellschaft. Beiträge des OECD/CERI-Regionalseminars für deutschsprachige Länder in Esslingen (Bundesrepublik Deutschland) vom 8.-12. Oktober 2001. Innsbruck, Wien, München u.a., S. 335-363.

Jäckel, Michael/Reinhardt, Jan D. (2003): Provokante Werbung unter dem Gesichtspunkt einer Ethik der Massenkommunikation. In: Debatin, Bernhard/Funiok, Rüdiger (Hg.): Kommunikations- und Medienethik. Konstanz, S. 203-218.

Karmasin, Matthias (1999): Medien. In: Korff, Wilhelm u. a. (Hg.): Handbuch der Wirtschaftsethik. Hg. im Auftr. der Görres-Gesellschaft. Band 4: Ausgewählte Handlungsfelder. Gütersloh, S. 351-381.

Luhmann, Niklas (1991): Paradigm lost. Über die ethische Reflexion der Moral. In: Luhmann, Niklas/Spaemann, Robert: Paradigm lost. Über die ethische 
Reflexion der Moral. Rede von Niklas Luhmann anläßlich der Verleihung des Hegel-Preises 1989. Laudatio von Robert Spaemann: Niklas Luhmanns Herausforderung der Philosophie. Frankfurt am Main, S. 9-48.

Marci-Boehncke, Gudrun/Rath, Matthias (2007): Medienkompetenz für ErzieherInnen. Ein Handbuch für die moderne Medienpraxis in der frühen Bildung. München.

Mieth, Dietmar (2004): Kleine Ethikschule. Freiburg, Basel, Wien.

Nolda, Sigrid (2002): Pädagogik und Medien. Eine Einführung. Stuttgart.

Schweer, Martin K./Schicha, Christian/Nieland, Jörg-Uwe (Hg.) (2002): Das Private in der öffentlichen Kommunikation. „Big Brother" und die Folgen. Köln.

Veith, Wemer (2002): Ethik der Rezeption. In: Hausmanninger, Thomas/ Bohrmann, Thomas (Hg.): Mediale Gewalt. Interdisziplinäre und ethische Perspektiven. München, S. 277-390.

Zimmer, Dieter E. (1970): Ein Medium kommt auf die Welt. Video-Kassetten und das neue multimediale Lernen. Hamburg. 